\title{
Study of the TP transformation via the inverted pendulum example
}

\author{
A. Wéber, M. Kuczmann
}

Széchenyi István University, Department of Automation

Egyetem tér 1., 9026, Gyốr

E-mail: andrea.weber.gyor@gmail.com

Submitted: 04/09/2020; Accepted: 16/11/2020; Published online: 09/12/2020

Abstract: This study decribes the Tensor Product (TP) based model construction through the nonlinear dynamic system of the inverted pendulum. It presents the steps of TP modeling, various weighting functions and the Linear Matrix Inequality (LMI) based approach. LMI control has been used to stabilize the nonlinear system. This study shows the quasi-Linear Parameter-Varying (qLPV) state-space modeling and the Higher-Order Singular Value Decomposition (HOSVD) based TP model transformation. Some research in this issue already exists, but only the furuta, rotary, single and parallel-type double pendulum have been examined. In this paper the TP model transformation of the inverted pendulum is analyzed in terms of stability.

Keywords: tensor product based control; nonlinear dynamic systems; linear matrix inequality; inverted pendulum

\section{Introduction}

Modeling and control of complicated nonlinear systems with multiple objectives are actually a challenge in control engineering. Many researchers investigate the $H_{2}, H_{\infty}$ [1], LQ-optimization, pole-placement [2] methods in control theory, which can be solved by LMI based approaches. Application of LMIs can reduce a wide species of problems appearing in systems and control engineering [3].

This paper introduces a qLPV model [4] of the inverted pendulum. The LPV systems [5] approach the nonlinear system in linear way, however the properties of 
the qLPV system are equivalent the nonlinear properties. It enables the representation of the nonlinear system without a complicated linearization method. LPV systems appear in the structure of Linear-Time Invariant (LTI) state-space representations. In this paper the LTI vertex system of the problem, the LMI design technique through the Parallel Distributed Compensation (PDC) and the HOSVD based TP transformation have been used. Close to Normalised (CNO) type weighting functions for an example have been applied MATLAB TP Toolbox [6]. TP transformation of pendulum has been investigated in some research for example rotary [7], furuta [8], single [9] [10] and parallel-type double pendulum [11]. This example is a good task to examine the TP model transformation and the stability of the nonlinear system.

The paper is organised as follows: in Section 2, the TP model transformation has introduced through the HOSVD based approach, the weighting functions and the qLPV model. Section 3 presents the LMI based controller design to obtain stable closed loop system based on the Lyapunov stability conditions. Due to nonlinearity, stable controller has been designed via LMI stability conditions. In Section 4, TP model transformation of the inverted pendulum has been presented, based on the Section 2-3.

\section{TP model transformation}

Control design of the TP model transformation was proposed by Baranyi et al. [12] [13]. The TP model structure [14] represents a multivariable tensor function. In fact, the TP model representation is a finite element convex polytopic representation [15] [16] [17]. In this paper the nonlinear system has approximated by the TP model transformation. The mathematical structure of this method is based on the HOSVD theory.

Singular value decomposition (SVD) [18] is a two dimensional matrix method used in the field of linear algebra. It is widespread in mathematics but this method is not suitable for analyzing multidimensional data. For multidimensional problems HOSVD methods [19] can be used. Multidimensional arrays or tensors represent data structures with more than two dimensions.

The HOSVD based computation extends its application to the qLPV model's continuos multivariable function. This produces numerically the TP model. TP transformation of HOSVD based approach generates the weighting funcions and the LTI vertex systems. The steps of transformating the TP model are the following [13]: 
- The given qLPV model must be discretized, which means the system matrix $\mathbf{S}(\mathbf{p})$ is converted numerically into a tensor representation, where $\mathbf{p}=\mathbf{p}(t) \in \Omega$ is a time-varying parameter vector within the closed hypercube $\Omega=\left[a_{1}, b_{1}\right] \times$ $\left[a_{2}, b_{2}\right] \times \ldots \times\left[a_{n}, b_{n}\right] \subset \mathbb{R}^{n}$. At first the problem space $\Omega$ is defined, which is a closed hypercube and the TP model is interpretable only in this space. $M$ denotes the discretization grid defined in $\Omega$.

- The TP model sctructure is extracted via the HOSVD structure to obtain the tensor product form.

- Weighting functions are defined. It is a computational method on HOSVD that can be converted numerically the qLPV model into that frame. The qLPV state-space representation is able to describe nonlinear systems because of combinating the LTI vertices and the nonlinear weighting functions $w_{i}=w_{i}(\mathbf{p}(t))$ i.e. weighting functions depend on the parameters. Types of the weighting functions are [13] [14]:

- (SN) Sum Normalised: the weighting function is SN if the sum of weighting functions for all $\mathbf{p}(t) \in \Omega$ is equal to 1 ;

- (NN) Non-Negative: values of the weighting functions are non- negative;

- (NO) Normalised: if the weighting function is SN and NN type and its largest value is 1 ;

- (CNO) Close to Normalised: if it is SN and NN type and its largest value is 1 or close to 1 ;

- (INO) Inverse Normalised: the weighting function is INO if its smallest value is 0 ;

- (RNO) Relaxed Normalised: the weighting function is RNO if its largest value is $\mathrm{SN}$ and NN type and that value is only between 0 and 1;

- (IRNO) Inverse Relaxed Normalised: the weighting function is IRNO if its smallest value is 0 and its largest value is $\mathrm{SN}$ and $\mathrm{NN}$ type and that value is only between 0 and 1 .

The conditions of convexity is not satisfied via HOSVD method [20] [21]. Thus, the convex hull created by the TP vertex system must be manipulated. The CNO type weighting function is used in this work to determine TP transformation of the inverted pendulum, see in Section 4. 
The continuous systems [22] are written by this form:

$$
\begin{aligned}
& \dot{\mathbf{x}}(t)=\mathbf{A}(\mathbf{p}(t)) \mathbf{x}(t)+\mathbf{B}(\mathbf{p}(t)) \mathbf{u}(t), \\
& \mathbf{y}(t)=\mathbf{C}(\mathbf{p}(t)) \mathbf{x}(t)+\mathbf{D}(\mathbf{p}(t)) \mathbf{u}(t),
\end{aligned}
$$

where $\mathbf{x}(t) \in \mathbb{R}^{n}$ is the state vector, $\mathbf{u}(t) \in \mathbb{R}^{m}$ is the input vector, $\mathbf{y}(t) \in \mathbb{R}^{q}$ is the output vector [14]. Equations (1) and (2) can be defined by combinating LTI systems. Thus, the representation can be written for instance:

$$
\begin{aligned}
& \dot{\mathbf{x}}(t)=\sum_{i=1}^{r} w_{i}(\mathbf{p}(t))\left(\mathbf{A}_{i} \mathbf{x}+\mathbf{B}_{i} \mathbf{u}\right), \\
& \mathbf{y}(t)=\sum_{i=1}^{r} w_{i}(\mathbf{p}(t))\left(\mathbf{C}_{i} \mathbf{x}+\mathbf{D}_{i} \mathbf{u}\right),
\end{aligned}
$$

where $\mathbf{A}_{\mathbf{i}}(t) \in \mathbb{R}^{n \times n}, \mathbf{B}_{\mathbf{i}}(t) \in \mathbb{R}^{n \times m}, \mathbf{C}_{\mathbf{i}}(t) \in \mathbb{R}^{q \times n}, \mathbf{D}_{\mathbf{i}}(t) \in \mathbb{R}^{q \times m}$ and $r$ is the vertex number. Equation (3) is the TP transformation. Weighting functions $w_{i}$ have two important properties that satisfy convexity [13]:

$$
\begin{aligned}
& w_{i}(\mathbf{p}(t)) \in[0,1], \\
& \sum_{i=1}^{r} w_{i}(\mathbf{p}(t))=1 .
\end{aligned}
$$

If parameter $\mathbf{p}(t)$ does not contain elements of $\mathbf{x}(t)$, it is an LPV system. If parameter $\mathbf{p}(t)$ contains elements of $\mathbf{x}(t)$, it is a qLPV model because of the nonlinearity. The qLPV model representation of state-space configuration (1) is:

$$
\mathbf{S}(\mathbf{x}(t))=\left[\begin{array}{ll}
\mathbf{A}(\mathbf{x}(t)) & \mathbf{B}(\mathbf{x}(t)) \\
\mathbf{C}(\mathbf{x}(t)) & \mathbf{D}(\mathbf{x}(t))
\end{array}\right]
$$


i.e.

$$
\left(\begin{array}{l}
\dot{\mathbf{x}} \\
\mathbf{y}
\end{array}\right)=\mathbf{S}(\mathbf{p})\left(\begin{array}{l}
\mathbf{x} \\
\mathbf{u}
\end{array}\right)
$$

\section{LMI based optimization}

In control science, application of LMI, especially in the field of dynamic system is related to Lyapunov [23]. This section presents the LMI based [2] control design approach. Many problems arise in control system design that are reduced by researchers to convex or quasi-convex optimization problem involving LMIs. LMIs are applied to solve many automation problems, optimization problems and system identification that are generally difficult to solve but it can be possible to solve by convex optimizing.

LMIs can solve in polynomial time. Many variety of systems and control problems can revise as LMI problems. These problems may be state-feedback synthesis, robustness design, $\mathrm{H}_{2}$ and $\mathrm{H}_{\infty}$ control [2]. It is reducible to convex problems. The variables are often matrices, therefore application of Lyapunov-inequality is the basic structure of the LMI method [31]:

$$
\mathbf{A}^{T} \mathbf{P A}-\mathbf{P} \prec 0,
$$

where $\mathbf{P}=\mathbf{P}^{T}$ is the variable. Finding $\mathbf{K}$ is the problem of control design. The closed-loop system is quadratically stable:

$$
[\mathbf{A}-\mathbf{B K}]^{T} \mathbf{P}[\mathbf{A}-\mathbf{B K}]-\mathbf{P} \prec 0,
$$

The above inequality is multiplied on the left and right via $\mathbf{P}^{-1}$, and $\mathbf{X}=\mathbf{P}^{-1}$ $\mathbf{M}=\mathbf{K X}$ :

$$
\mathbf{P}^{-1}[\mathbf{A}-\mathbf{B K}]^{T} \mathbf{P}[\mathbf{A}-\mathbf{B K}] \mathbf{P}^{-1}-\mathbf{P}^{-1} \mathbf{P P}^{-1} \prec 0,
$$


i.e.

$$
\mathbf{X}[\mathbf{A}-\mathbf{B K}]^{T} \mathbf{X}^{-1}[\mathbf{A}-\mathbf{B K}] \mathbf{X}-\mathbf{X} \prec 0
$$

finally

$$
\mathbf{X}-[\mathbf{A X}-\mathbf{B M}]^{T} \mathbf{X}^{-1}[\mathbf{A X}-\mathbf{B M}] \succ 0 .
$$

It can be transformed into the LMI form:

$$
\left[\begin{array}{cc}
\mathbf{X} & (\mathbf{A X}-\mathbf{B M})^{T} \\
(\mathbf{A X}-\mathbf{B M}) & \mathbf{X}
\end{array}\right] \succ 0 .
$$

This form is defined by Schur complements. Defining the Schur complements [2] of a partitioned A matrix can be written as:

$$
\mathbf{A}=\left[\begin{array}{ll}
A_{11} & A_{12} \\
A_{21} & A_{22}
\end{array}\right]
$$

For example if $A_{11}$ is nonsingular, $A_{22}-A_{21} A_{11}^{-1} A_{12}$ is the Schur complement of $A_{11}$. It can be denoted by $S_{c h}\left(A_{11}\right)$. Thus, matrix $\mathbf{A}$ can be written as:

$$
\mathbf{A}=\left[\begin{array}{cc}
A_{11} & 0 \\
0 & S_{c h}\left(A_{11}\right)
\end{array}\right]
$$

\subsection{Presenting LMI based approach}

PDC is a model based design procedure that was suggested by Tanaka and Wang [24]. They designed for Takagi-Sugeno (TS) fuzzy decision [31] models so their procedure was to develop a fuzzy controller from a TS fuzzy model. Combination of LMI optimization and PDC framework can be accomplished with convex optimization. The PDC design structures are defined one LTI feedback gain $\mathbf{k}$ to all LTI vertex systems. 
Therefore, the TP controller is:

$$
\mathbf{u}(t)=-\sum_{i=1}^{r} w_{i}(\mathbf{p}(t)) \mathbf{k}_{i} \mathbf{x}(t)=-\left(\sum_{i=1}^{r} w_{i}(\mathbf{p}(t)) \mathbf{k}_{i}\right) \mathbf{x}(t)=-\mathbf{k}^{T} \mathbf{x}(t) .
$$

Substituting (17) into (3) the following representation is obtained:

$$
\begin{gathered}
\dot{\mathbf{x}}(t)=\sum_{i=1}^{r} w_{i}\left[\mathbf{A}_{i} \mathbf{x}(t)+\mathbf{B}_{i}\left(-\sum_{j=1}^{r} w_{j} \mathbf{k}_{j} \mathbf{x}(t)\right)\right] \\
\dot{\mathbf{x}}(t)=\sum_{i=1}^{r} \sum_{j=1}^{r} w_{i} w_{j}\left[\mathbf{A}_{i}-\mathbf{B}_{i} \mathbf{k}_{j}\right] \mathbf{x}(t)
\end{gathered}
$$

where $i, j=1 \ldots r, r$ is the total number of LTI systems. It can be rewritten into this form:

$$
\dot{\mathbf{x}}(t)=\sum_{i=1}^{r} w_{i} w_{i}\left[\mathbf{A}_{i}-\mathbf{B}_{i} \mathbf{k}_{i}\right] \mathbf{x}(t)+2 \sum_{i=1}^{r} \sum_{i<j}^{r} w_{i} w_{j} \mathbf{G}_{i j} \mathbf{x}(t),
$$

where $\mathbf{G}$ is:

$$
\mathbf{G}_{i j}=\frac{\left[\mathbf{A}_{i}-\mathbf{B}_{i} \mathbf{k}_{j}\right]+\left[\mathbf{A}_{j}-\mathbf{B}_{j} \mathbf{k}_{i}\right]}{2} .
$$

\subsection{The Lyapunov theory}

The LMI based approach began with Lyapunov [23] based stability conditions. In the case of nonlinear dynamic systems, stability analysis methods developed for linear systems, are not applicable. The Lyapunov methods are used to get suifficent conditions for stability of the equilibrium point of a linear system.

Consider a system $\dot{\mathbf{x}}=\mathbf{f}(\mathbf{x}, t)$, where $\mathbf{f}$ is continuos. To study the stability of nonlinear dynamic systems, the Lyapunov theorem can be applied. If there exists a function $V(\mathbf{x}, t)$ what can be derived around an equilibrium point $\mathbf{x}=0$ and satisfies the following conditions: 
- $V(\mathbf{0}, t)=0$ and $V(\mathbf{x}, t)>0$ if $V(\mathbf{x}, t)$ is a positive defitine function,

- $\dot{V}(\mathbf{x}, t) \leq 0$, i.e. $\dot{V}(\mathbf{x}, t)$ is a negative semidefinite function,

- $\dot{V}(\mathbf{x}, t)<0$, i.e. $\dot{V}(\mathbf{x}, t)$ is a negative definite function.

If first and second conditions are existed, then the system is stable, and if the first and third conditions are existed the system is asymptotically stable. The function $V$ in Lyapunov theorem is the energy function assigned to the system.

Consider a system $\dot{\mathbf{x}}=\mathbf{f}(\mathbf{x}, \mathbf{u})$ with control inputs $u$, where $\mathbf{x}(t) \in \mathbb{R}^{n}, \mathbf{u}(t) \in \mathbb{R}^{m}$. Consider the candidate Lyapunov function $V(\mathbf{x})$ for every fixed $\mathbf{x} \neq 0$ there exists and acceptable value $\mathbf{u}$ for the control in such a way as to:

$$
\nabla V(\mathbf{x}) \cdot \mathbf{f}(\mathbf{x}, \mathbf{u})<0
$$

Regard the following candidate Lyapunov function [31] $V(\mathbf{x}(t))=\mathbf{x}^{T}(t) \mathbf{P} \mathbf{x}(t)$ where $\mathbf{P} \succ 0$, thus:

$$
\begin{aligned}
\dot{\mathbf{V}}(\mathbf{x}(t)) & =\sum_{i=1}^{r} \sum_{j=1}^{r} w_{i} w_{j} \mathbf{x}^{T}(t)\left[\left(\mathbf{A}_{i}-\mathbf{B}_{i} \mathbf{k}_{j}\right)^{T} \mathbf{P}+\mathbf{P}\left(\mathbf{A}_{i}-\mathbf{B}_{i} \mathbf{k}_{j}\right)\right] \mathbf{x}(t) \\
& =\sum_{i=1}^{r} w_{i}^{2} \mathbf{x}^{T}(t)\left[\mathbf{G}_{i i}^{T} \mathbf{P}+\mathbf{P G}_{i i}\right] \mathbf{x}(t)+\sum_{i=1}^{r} \sum_{i<j} 2 w_{i} w_{j} \mathbf{x}^{T}(t) \\
& \times\left[\left(\frac{\mathbf{G}_{i j}+\mathbf{G}_{j i}}{2}\right)^{T} \mathbf{P}+\mathbf{P}\left(\frac{\mathbf{G}_{i j}+\mathbf{G}_{j i}}{2}\right)\right] \mathbf{x}(t) .
\end{aligned}
$$

\subsection{Stable controller design with LMI based stability conditions}

Consider the stability of an open-loop system via Lyapunov stability concept, where the equilibrium of TP system (19) via $\mathbf{u}(t)=0$ is globally asymptotically stable, then there is a common positive definite matrix $\mathbf{P}$ for instance

$$
\mathbf{A}_{i}^{T} \mathbf{P}+\mathbf{P} \mathbf{A}_{i} \prec 0
$$


for $\forall i=1, \cdots, r$. Examine stability of an closed-loop system, as:

$$
\begin{gathered}
\dot{\mathbf{x}}(t)=\sum_{i=1}^{r} \sum_{j=1}^{r} w_{i} w_{j}\left(\mathbf{A}_{i}-\mathbf{B}_{i} \mathbf{K}_{j}\right) \mathbf{x}(t), \\
\dot{\mathbf{x}}(t)=\sum_{i=1}^{r} w_{i} w_{i}\left[\mathbf{A}_{i}-\mathbf{B}_{i} \mathbf{K}_{i}\right] \mathbf{x}(t)+2 \sum_{i=1}^{r} \sum_{i<j}^{r} w_{i} w_{j} \mathbf{G}_{i j} \mathbf{x}(t),
\end{gathered}
$$

Let us use the following notations:

$$
\begin{array}{r}
\mathbf{G}_{i j}=\mathbf{A}_{i}-\mathbf{B}_{i} \mathbf{K}_{j}, \\
\mathbf{G}_{j i}=\mathbf{A}_{j}-\mathbf{B}_{j} \mathbf{K}_{i},
\end{array}
$$

where $i=1, \cdots, r$. The equilibrium point of the TP system (9) is globally asymptotically stable if there is a common positive definite matrix $\mathbf{P}$ :

$$
\mathbf{G}_{i i}^{T} \mathbf{P}+\mathbf{P G}_{i i} \prec 0
$$

and

$$
\left(\frac{\mathbf{G}_{i j}+\mathbf{G}_{j i}}{2}\right)^{T} \mathbf{P}+\mathbf{P}\left(\frac{\mathbf{G}_{i j}+\mathbf{G}_{j i}}{2}\right) \preceq 0 .
$$

Multiplying inequelity (29) on the left and right by $\mathbf{P}^{-1}$ and denote $\mathbf{X}=\mathbf{P}^{-1}$ and $\mathbf{M}_{\mathbf{i}}=\mathbf{K}_{\mathbf{i}} \mathbf{X}$ :

$$
\left(\mathbf{P}^{-1} \mathbf{A}_{i} \mathbf{P}-\mathbf{P}^{-1} \mathbf{B}_{i} \mathbf{K}_{i} \mathbf{P}\right)^{T}+\left(\mathbf{P}^{-1} \mathbf{P} \mathbf{A}_{i}-\mathbf{P}^{-1} \mathbf{P} \mathbf{B}_{i} \mathbf{K}_{i}\right) \prec 0,
$$


finally

$$
\begin{aligned}
& \left(\mathbf{P}^{-1} \mathbf{A}_{i} \mathbf{P} \mathbf{P}^{-1}-\mathbf{P}^{-1} \mathbf{B}_{i} \mathbf{K}_{i} \mathbf{P} \mathbf{P}^{-1}\right)^{T} \\
& +\left(\mathbf{P}^{-1} \mathbf{P} \mathbf{A}_{i} \mathbf{P}^{-1}-\mathbf{P}^{-1} \mathbf{P} \mathbf{B}_{i} \mathbf{K}_{i} \mathbf{P}^{-1}\right) \prec 0,
\end{aligned}
$$

Substituting $\mathbf{X}=\mathbf{P}^{-1}$ and $\mathbf{M}_{\mathbf{i}}=\mathbf{K}_{\mathbf{i}} \mathbf{X}$, then:

$$
-\mathbf{X} \mathbf{A}_{i}^{T}-\mathbf{A}_{i} \mathbf{X}+\mathbf{X K}_{i}^{T} \mathbf{B}_{i}^{T}+\mathbf{B}_{i} \mathbf{K}_{i} \mathbf{X} \succ 0
$$

the following LMI stability condition is got:

$$
-\mathbf{X} \mathbf{A}_{i}^{T}-\mathbf{A}_{i} \mathbf{X}+\mathbf{M}_{i}^{T} \mathbf{B}_{i}^{T}+\mathbf{B}_{i} \mathbf{M}_{i} \succ 0 .
$$

Equations (27) and (28) are applied, and denote $\mathbf{X}=\mathbf{P}^{-1}$ and $\mathbf{M}_{\mathbf{i}}=\mathbf{K}_{\mathbf{i}} \mathbf{X}$ :

$$
\begin{aligned}
& \left(\frac{\mathbf{A}_{i}-\mathbf{B}_{i} \mathbf{K}_{j}+\mathbf{A}_{j}-\mathbf{B}_{j} \mathbf{K}_{i}}{2}\right)^{T} \mathbf{P}+\mathbf{P} \\
& \left(\frac{\mathbf{A}_{i}-\mathbf{B}_{i} \mathbf{K}_{j}+\mathbf{A}_{j}-\mathbf{B}_{j} \mathbf{K}_{i}}{2}\right) \preceq 0,
\end{aligned}
$$

then multiplying inequelity (30) on the left and right by $\mathbf{P}^{-1}$, then the following equation is got:

$$
\begin{aligned}
& \left(\frac{\mathbf{A}_{i} \mathbf{P}-\mathbf{B}_{i} \mathbf{K}_{j} \mathbf{P}+\mathbf{A}_{j} \mathbf{P}-\mathbf{B}_{j} \mathbf{K}_{i} \mathbf{P}}{2}\right)^{T} \\
& +\left(\frac{\mathbf{P A}_{i}-\mathbf{P B}_{i} \mathbf{K}_{j}+\mathbf{P} \mathbf{A}_{j}-\mathbf{P} \mathbf{B}_{j} \mathbf{K}_{i}}{2}\right) \preceq 0,
\end{aligned}
$$




$$
\begin{gathered}
\left(\frac{\mathbf{P}^{-1} \mathbf{A}_{i} \mathbf{P}-\mathbf{P}^{-1} \mathbf{B}_{i} \mathbf{K}_{j} \mathbf{P}+\mathbf{P}^{-1} \mathbf{A}_{j} \mathbf{P}-\mathbf{P}^{-1} \mathbf{B}_{j} \mathbf{K}_{i} \mathbf{P}}{2}\right)^{T} \\
+\left(\frac{\mathbf{P}^{-1} \mathbf{P} \mathbf{A}_{i}-\mathbf{P}^{-1} \mathbf{P} \mathbf{B}_{i} \mathbf{K}_{j}+\mathbf{P}^{-1} \mathbf{P} \mathbf{A}_{j}-\mathbf{P}^{-1} \mathbf{P} \mathbf{B}_{j} \mathbf{K}_{i}}{2}\right) \preceq 0 \\
\left(\frac{\mathbf{P}^{-1} \mathbf{A}_{i} \mathbf{P} \mathbf{P}^{-1}-\mathbf{P}^{-1} \mathbf{B}_{i} \mathbf{K}_{j} \mathbf{P} \mathbf{P}^{-1}+\mathbf{P}^{-1} \mathbf{A}_{j} \mathbf{P} \mathbf{P}^{-1}-\mathbf{P}^{-1} \mathbf{B}_{j} \mathbf{K}_{i} \mathbf{P} \mathbf{P}^{-1}}{2}\right)^{T} \\
+\left(\frac{\mathbf{P}^{-1} \mathbf{P} \mathbf{A}_{i} \mathbf{P}^{-1}-\mathbf{P}^{-1} \mathbf{P} \mathbf{B}_{i} \mathbf{K}_{j} \mathbf{P}^{-1}+\mathbf{P}^{-1} \mathbf{P} \mathbf{A}_{j} \mathbf{P}^{-1}-\mathbf{P}^{-1} \mathbf{P} \mathbf{B}_{j} \mathbf{K}_{i} \mathbf{P}^{-1}}{2}\right) \preceq 0
\end{gathered}
$$

Substituting $\mathbf{X}=\mathbf{P}^{-1}$ and $\mathbf{M}_{\mathbf{i}}=\mathbf{K}_{\mathbf{i}} \mathbf{X}$ :

$$
\begin{aligned}
& \left(\frac{\mathbf{X} \mathbf{A}_{i}-\mathbf{X B}_{i} \mathbf{K}_{j}+\mathbf{X} \mathbf{A}_{j}-\mathbf{X} \mathbf{B}_{j} \mathbf{K}_{i}}{2}\right)^{T} \\
& +\left(\frac{\mathbf{A}_{i} \mathbf{X}-\mathbf{B}_{i} \mathbf{K}_{j} \mathbf{X}+\mathbf{A}_{j} \mathbf{X}-\mathbf{B}_{j} \mathbf{K}_{i} \mathbf{X}}{2}\right) \preceq 0,
\end{aligned}
$$

$-\mathbf{X} \mathbf{A}_{i}^{T}-\mathbf{A}_{i} \mathbf{X}-\mathbf{X} \mathbf{A}_{j}^{T}-\mathbf{A}_{j} \mathbf{X}+\mathbf{X} \mathbf{K}_{j}^{T} \mathbf{B}_{i}^{T}+\mathbf{B}_{i} \mathbf{K}_{j} \mathbf{X}+\mathbf{X} \mathbf{K}_{i}^{T} \mathbf{B}_{j}^{T}+\mathbf{B}_{j} \mathbf{K}_{i} \mathbf{X} \succ 0$,

after this, another LMI stability condition is got:

$$
-\mathbf{X} \mathbf{A}_{i}^{T}-\mathbf{A}_{i} \mathbf{X}-\mathbf{X} \mathbf{A}_{j}^{T}-\mathbf{A}_{j} \mathbf{X}+\mathbf{M}_{j}^{T} \mathbf{B}_{i}^{T}+\mathbf{B}_{i} \mathbf{M}_{j}+\mathbf{M}_{i}^{T} \mathbf{B}_{j}^{T}+\mathbf{B}_{j} \mathbf{M}_{i} \succeq 0 .
$$

Equations (34) and (41) are the LMI stability conditions and these are applied for the inverted pendulum example by equations (66) and (67). 


\section{TP transformation of the inverted pendulum}

The center of mass of the inverted pendulum [25] is above its pivot point. Control designing of this nonlinear system has been the subject of many studies and researchers in the recent period [26] [27] [28].

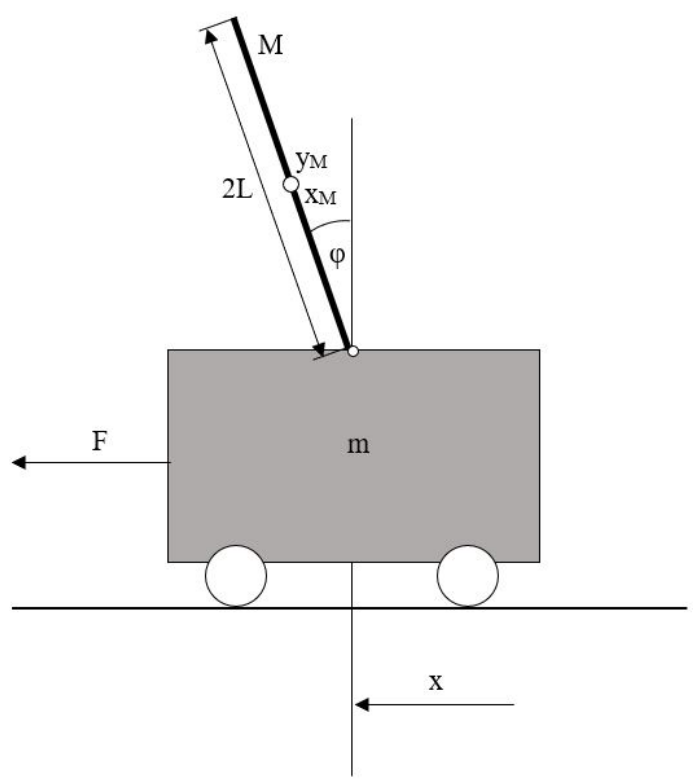

Figure 1. Inverted Pendulum

\subsection{Modeling of inverted pendulum}

The illustration of the inverted pendulum installed on a cart is given in Fig 1. The lenght of the rod on the car is $2 L=0.35$, the mass of the rod is $M=1.2$, the mass of the cart $m=0.2$ in a coherent unit system SI and the generalized coordinates are $x$ and $\varphi$. The system equations of the nonlinear system are written by Euler-Lagrange 
equation [29]:

$$
\frac{d}{d t} \frac{\partial K}{\partial \dot{q}_{i}}-\frac{\partial K}{\partial q_{i}}+\frac{\partial P}{\partial q_{i}}=\tau_{i}
$$

where $K$ is the kinetic energy, $P$ is the potential energy, $q_{i}$ is the generalized coordinate and $\tau_{i}$ is the generalized force. The moment of inertia of the rod belonging to the center of mass is $\Theta=\frac{M L^{2}}{3}$. The center of gravity of the rod mass is given by the coordinates $x_{M}=x+L S_{\varphi}$ and $y_{M}=L C_{\varphi}$ and the velocity is $v_{M}$. Let's denote $S_{\varphi}=\sin (\varphi)$ and $C_{\varphi}=\cos (\varphi)$ [29] [26] [32]. After derivating $x_{M}$ and $y_{M}$, the following results can be got:

$$
\begin{gathered}
\dot{x}_{M}=\dot{x}+\dot{\varphi} L C_{\varphi}, \\
\dot{y}_{M}=-\dot{\varphi} L S_{\varphi}, \\
v_{M}^{2}=\dot{x}_{M}^{2}+\dot{y}_{M}^{2}=\dot{x}^{2}+2 L C_{\varphi} \dot{x} \dot{\varphi}+L^{2} \dot{\varphi}^{2} .
\end{gathered}
$$

Determining kinetic energy and potential energy as follows:

$$
\begin{gathered}
K=\frac{1}{2} m \dot{x}^{2}+\frac{1}{2} M v_{M}^{2}+\frac{1}{2} \Theta \dot{\varphi}^{2} \\
=\frac{1}{2} m \dot{x}^{2}+\frac{1}{2} M\left(\dot{x}^{2}+2 L C_{\varphi} \dot{x} \dot{\varphi}+L^{2} \dot{\varphi}^{2}\right)+\frac{1}{2} \Theta \dot{\varphi}^{2}, \\
P=M g L C_{\varphi} .
\end{gathered}
$$

After obtaining the partial derivatives of $K$ and $P$, and applying mathematical manipulations, the following equations are given:

$$
\frac{d}{d t} \frac{\partial K}{\partial \dot{x}}=(m+M) \ddot{x}+M L C_{\varphi} \ddot{\varphi}-M L S_{\varphi} \dot{\varphi}^{2},
$$


A. Wéber and M. Kuczmann - Acta Technica Jaurinensis, Vol. 14, No. 1, pp. 1-23, 2021

$$
\frac{d}{d t} \frac{\partial K}{\partial \dot{\varphi}}=M L C_{\varphi} \ddot{x}-M L S_{\varphi} \dot{x} \dot{\varphi}+\left(\Theta+M L^{2}\right) \ddot{\varphi}
$$

Using Euler-Lagrange equation (42), the following equations are got:

$$
\begin{gathered}
\frac{d}{d t} \frac{\partial K}{\partial \dot{x}}-\frac{\partial K}{\partial x}+\frac{\partial P}{\partial x}=F, \\
\frac{d}{d t} \frac{\partial K}{\partial \dot{\varphi}}-\frac{\partial K}{\partial \varphi}+\frac{\partial P}{\partial \varphi}=0,
\end{gathered}
$$

i.e.

$$
\begin{gathered}
(M+m) \ddot{x}+M L C_{\varphi} \ddot{\varphi}-M L S_{\varphi} \dot{\varphi}^{2}=F \\
M L C_{\varphi} \ddot{x}+\left(\Theta+M L^{2}\right) \ddot{\varphi}-M g L S_{\varphi}=0 .
\end{gathered}
$$

After some mathematical manipulations the following equations are given:

$$
\begin{gathered}
\ddot{x}=\frac{F-M L C_{\varphi} \ddot{\varphi}+M L S_{\varphi} \dot{\varphi}^{2}}{m+M}, \\
\ddot{\varphi}=\frac{g S_{\varphi}-\frac{C_{\varphi}}{m+M}\left(F+M L S_{\varphi} \dot{\varphi}^{2}\right)}{L\left(\frac{4}{3}-\frac{M C_{\varphi}^{2}}{m+M}\right)} .
\end{gathered}
$$

Then the following state-space representation is defined:

$$
\begin{gathered}
\dot{x}_{1}=x_{2}, \\
\dot{x}_{2}=\frac{\frac{4}{3} M L \sin \left(x_{3}\right) x_{4}^{2}-M g \cos \left(x_{3}\right) \sin \left(x_{3}\right)+\frac{4}{3} F}{\frac{4}{3}(m+M)-M \cos ^{2}\left(x_{3}\right)}, \\
\dot{x}_{3}=x_{4},
\end{gathered}
$$




$$
\dot{x}_{4}=\frac{M L \sin \left(x_{3}\right) \cos \left(x_{3}\right) x_{4}^{2}+\cos \left(x_{3}\right) F-(m+M) g \sin \left(x_{3}\right)}{M L \sin ^{2}\left(x_{3}\right)-\frac{4}{3}(m+M) L},
$$

where the state-space variables are $x_{1}=x, x_{2}=\dot{x}, x_{3}=\varphi, x_{4}=\dot{\varphi}$.

\subsection{TP transformation}

It is an important purpose of transforming TP to convert the given state-space model (1) into a convex TP model. In this model the LTI systems create a tight convex hull [30]. Consider the following state-space structure:

$$
\dot{\mathbf{x}}(t)=\mathbf{A}(\mathbf{x}) \mathbf{x}+\mathbf{B}(\mathbf{x}) u,
$$

Elements of matrix $\mathbf{A}(\boldsymbol{x})$ (61) and vector $\mathbf{B}(\mathbf{x})$ (62) depend on only the state variables $x_{3}$ and $x_{4}$, i.e. nonlinearity is caused by $x_{3}$ and $x_{4}$. For TP model transfromation the transformation space $\Omega=[(-45 / 180) \pi,(45 / 180) \pi] \times[(-45 / 180) \pi,(45 / 180) \pi]$ is defined and discretized by $M_{1} \times M_{2}=136 \times 136$ grid points. The components of matrix $\mathbf{A}(\boldsymbol{x})$ and vector $\mathbf{B}(\mathbf{x})$ have been defined numerically and they are stored in a four dimensional tensor $S \in R^{136 \times 136 \times 4 \times 5}$,

$$
\mathbf{A}(\boldsymbol{x})=\left[\begin{array}{cccc}
0 & 1 & 0 & 0 \\
0 & 0 & \frac{-M g \cos \left(x_{3}\right)}{\frac{4}{3}(m+M)-M \cos ^{2}\left(x_{3}\right)} \frac{\sin \left(x_{3}\right)}{x_{3}} & \frac{\frac{4}{3} M L \sin \left(x_{3}\right)}{\frac{4}{3}(m+M)-M \cos ^{2}\left(x_{3}\right)} x_{4} \\
0 & 0 & 0 & 1 \\
0 & 0 & -\frac{(m+M) g}{M L \sin ^{2}\left(x_{3}\right)-\frac{4}{3}(m+M) L} \frac{\sin \left(x_{3}\right)}{x_{3}} & \frac{M L \sin \left(x_{3}\right) \cos \left(x_{3}\right)}{M L \sin \left(x_{3}\right)-\frac{4}{3}(m+M) L} x_{4}
\end{array}\right],
$$

$$
\mathbf{B}(\mathbf{x})=\left[\begin{array}{c}
0 \\
\frac{4}{3} \\
\frac{4}{3}(m+M)-M \cos ^{2}\left(x_{3}\right) \\
\frac{\cos \left(x_{3}\right)}{M L \sin ^{2}\left(x_{3}\right)-\frac{4}{3}(m+M) L}
\end{array}\right]
$$

Applying HOSVD, the singular values are $\sigma_{1}=1909, \sigma_{2}=201, \sigma_{3}=23, \sigma_{4}=2$ for the variable $x_{3}$ and $\sigma_{1}=1920, \sigma_{2}=23$ for the variable $x_{4}$. After using HOSVD 
on $\mathbf{S}(\mathbf{x}(t))$ and CNO type weighting function, the following TP model transformation describes inverted pendulum system:

$$
\dot{\mathbf{x}} \cong \sum_{i_{1}=1}^{r_{1}} \sum_{i_{2}=1}^{r_{2}} w_{i_{1}}\left(x_{3}\right) w_{i_{2}}\left(x_{4}\right)\left(\mathbf{A}_{i_{1}, i_{2}} \mathbf{x}+\mathbf{B}_{i_{1}, i_{2}} u\right),
$$

where $w_{i_{1}}\left(x_{3}\right)$ and $w_{i_{2}}\left(x_{4}\right)$ are the CNO type weighting functions, $\mathbf{A}_{i_{1}, i_{2}}$ and $\mathbf{B}_{i_{1}, i_{2}}$ are state independent system matrix and vector. This nonlinear system is possible to be approximated by the combinations of $r_{1} r_{2}$ LTI systems. It can be written as follows:

$$
\begin{gathered}
\dot{\mathbf{x}} \cong \sum_{i_{1}=1}^{5} \sum_{i_{2}=1}^{2} w_{i_{1}}\left(x_{3}\right) w_{i_{2}}\left(x_{4}\right)\left(\mathbf{A}_{i_{1}, i_{2}} \mathbf{x}+\mathbf{B}_{i_{1}, i_{2}} u\right), \\
\dot{\mathbf{x}} \cong \sum_{i=1}^{r} w_{i}\left(x_{3}, x_{4}\right)\left(\mathbf{A}_{i} \mathbf{x}+\mathbf{B}_{i} u\right) .
\end{gathered}
$$

where $r=r_{1} r_{2}$ is the number of LTI systems via $5 \times 2=10$ LTI vertex models. Weighting functions $w_{i_{1}}\left(x_{3}\right)$ and $w_{i_{2}}\left(x_{4}\right)$ are shown in Fig. 2-3. Thus, the system is decomposed in angular velocity $\dot{\varphi}$ into 2 weighting functions, and in angular position $\varphi$ into 5 weighting functions. 
A. Wéber and M. Kuczmann - Acta Technica Jaurinensis, Vol. 14, No. 1, pp. 1-23, 2021

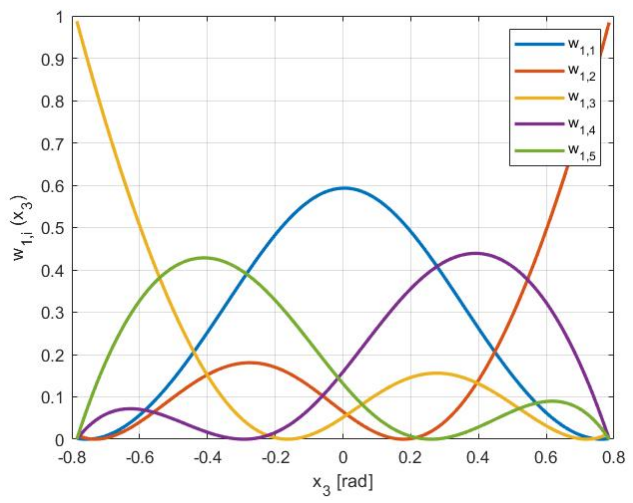

Figure 2. CNO type weighting functions for $x_{3}$

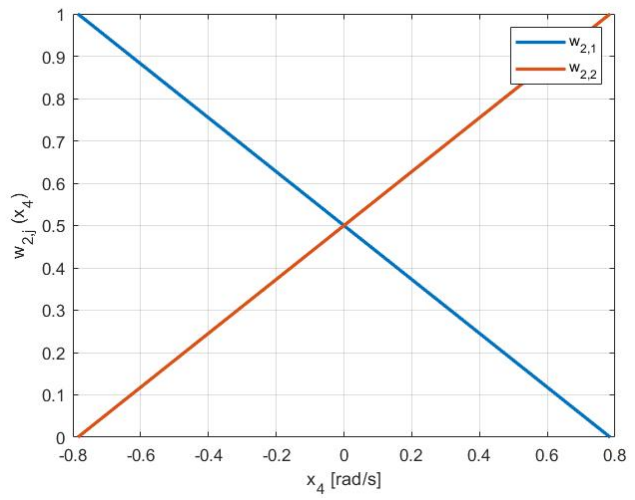

Figure 3. CNO type weighting functions for $x_{4}$ 
The controller vector $\mathbf{k}_{i}$ can be numerically defined by LMI. Using stability conditions (31) and (41), determining asymptotic stability of the system. Thus, the stabilization controller can be found:

$$
-\mathbf{X} \mathbf{A}_{i}^{T}-\mathbf{A}_{i} \mathbf{X}+\mathbf{M}_{i}^{T} \mathbf{B}_{i}^{T}+\mathbf{B}_{i} \mathbf{M}_{i} \succ 0
$$

$$
-\mathbf{X} \mathbf{A}_{i}^{T}-\mathbf{A}_{i} \mathbf{X}-\mathbf{X} \mathbf{A}_{j}^{T}-\mathbf{A}_{j} \mathbf{X}+\mathbf{M}_{j}^{T} \mathbf{B}_{i}^{T}+\mathbf{B}_{i} \mathbf{M}_{j}+\mathbf{M}_{i}^{T} \mathbf{B}_{j}^{T}+\mathbf{B}_{j} \mathbf{M}_{i} \succeq 0,
$$

$$
\mathbf{k}_{i}=\mathbf{M}_{i} \mathbf{X}^{-1}
$$

where $i=1, \cdots, r$ and $j=i+1, \cdots, r$, and $r$ is the number of LTI vertex systems.

The control signal is shown in Fig. 6. and state variables $x_{1}$ and $x_{3}$ are shown in Fig. 4-5. The initial condition is $\mathbf{x}(0)=[0.3,0,0,0]$.

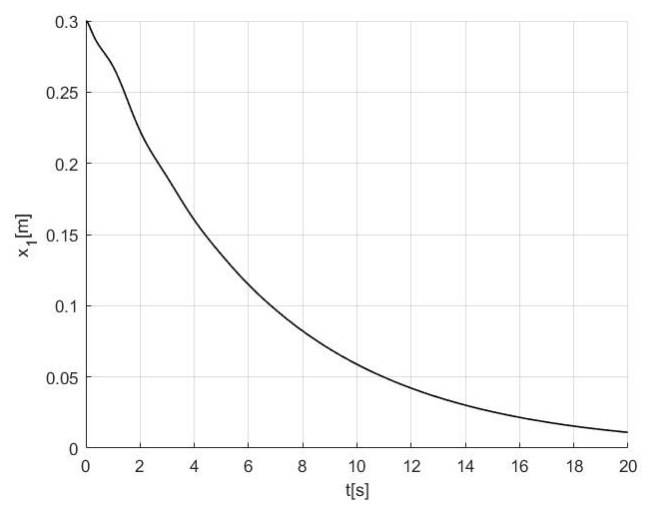

Figure 4. State variable $x_{1}$

It can be seen in Fig. 4-6. that functions $x_{3}$ and $u$ return to a steady state after a few oscillations. The system get into the stable position around 10 seconds. This will result that LMI approach should improve to stabilize better this nonlinear system. 
A. Wéber and M. Kuczmann - Acta Technica Jaurinensis, Vol. 14, No. 1, pp. 1-23, 2021

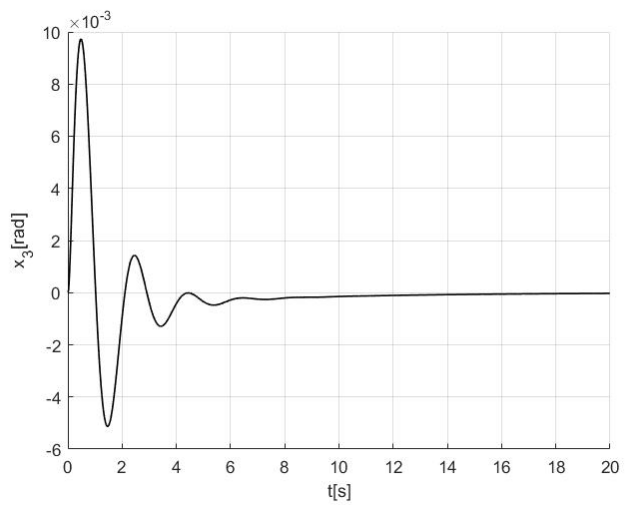

Figure 5. State variable $x_{3}$

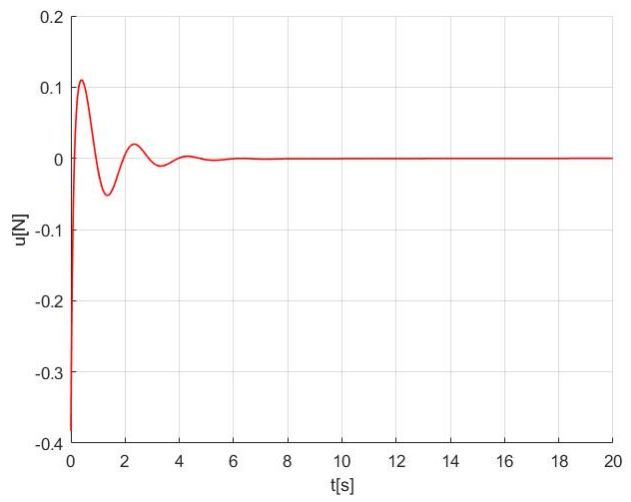

Figure 6. Control signal u 


\section{Conclusion}

This paper describes a study of the HOSVD based TP transformation with CNO type weighting functions using LMI solver and a solution to a problem by applying these methods through an inverted pendulum example. In this example there are 10 LTI vertex systems of the qLPV model. This paper shows an example to analyse global asymptotic stability. Therefore in the future the decay rate for the inverted pendulum and $H_{2}, H_{\infty}$ controllers will be analysed.

\section{References}

[1] S. Iles, F. Kolonic, J. Matusko, Linear Matrix Inequalities Based $H_{\infty}$ Control of Gantry Crane using Tensor Product Transformation, Proceedings of the 18th International Conference on ProcessControl, Tatranská Lomnica, Slovakia, 2011, pp. 92-99.

[2] D. Guang-Ren, Y. Hai-Hua, LMIs in Control Systems: Analysis, Design and Applications, 1st Edition, CRC Press, Taylor and Francis Group, Boca Raton, 2013.

doi: https://doi.org/10.1201/b15060

[3] X. Liu, X. Xin, Z. Li, Z. Chen, Near Optimal Control Based on the TensorProduct Technique, IEEE Transactions on Circuits and Systems II: Express Briefs 64 (5) (2017) pp. 560-564.

doi: https://doi.org/10.1109/TCSII.2016.2592986

[4] A. Szollosi, P. Baranyi, Influence of the Tensor Product model representation of qLPV models on the feasibility of Linear Matrix Inequality, Asian Journal of Control 18 (4) (2015) pp. 1328-1342.

[5] B. Lantos, Theory and Design of Control Systems III., Academic Press, Budapest, 2018.

[6] Sz. Nagy, Z. Petres, P. Baranyi, TP tool - A MATLAB toolbox for TP model transformation, 8th International Symposium of Hungarian Researchers on Computational Intelligence and Informatics, CINTI (2007), Budapest, Hungary pp. 483-495.

[7] S. Iles, J. Matusko, F. Kolonic, Furuta Pendulum - a Tensor Product Modelbased Design Approach Case Study, 2011 Proceedings of the 34th International Convention MIPRO, Opatija, Croatia, 2011. 
[8] P. Grof, Y. Yam, TP transformation based control of rotary pendulumy, IEEE International Conference on Systems, Man, and Cybernetics (2015) pp. 26202625.

doi: https://doi.org/10.1109/SMC.2015.458

[9] J. Matusko, V. Lesic, F. Kolonic, S. Iles, Tensor product based control of the Single Pendulum Gantry process with stable neural network based friction compensation, Advanced Intelligent Mechatronics (AIM), 2011 IEEE/ASME International Conference, Budapest.

doi: https://doi.org/10.1109/AIM.2011.6027152

[10] F. Kolonic, A. Poljugan, Experimental Control Design by TP Model Transformation, 2006 IEEE International Conference on Mechatronics, Budapest, Hungary, 2006.

doi: https://doi.org/10.1109/ICMECH.2006.252605

[11] Sz. Nagy, Z. Petres, P. Baranyi, TP model transformation based controller design for the parallel-type double inverted pendulum, 2008 IEEE International Conference on Fuzzy Systems (IEEE World Congress on Computational Intelligence), Hong Kong, China, 2008.

doi: https://doi.org/10.1109/FUZzY.2008.4630551

[12] P. Baranyi, TP- Model Transformation Based Control Design Frameworks, Control Engineering, Springer Book, 2016.

ISBN: 978-3-319-19605-3

[13] P. Baranyi, Y. Yam, P. Várlaki, Tensor Product Model Transformation in Polytopic Model Based Control, 1st Edition, Automation and Control Engineering, CRC Press, Taylor and Francis Group, 2018.

doi: https://doi.org/10.1201/9781315218045

[14] P. Baranyi, Extracting LPV and qLPV Structures from State-Space Functions: A TP Model Transformation Based Framework, IEEE Transactions on Fuzzy Systems 28 (3) (2017) pp. 499-509.

doi: https://doi.org/10.1109/TFUZZ.2019.2908770

[15] P. Korondi, Tensor Product Model Transformation-based Sliding Surface Design, Acta Polytechnica Hungarica 3 (4) (2006) pp. 23-35.

[16] F. Kolonic, A. Poljugan, I. Petrovic, Tensor Product Model Transformationbased Controller Design for Gantry Crane Control System-an Application Approach, Acta Polytechnica Hungarica 3 (4) (2006) pp. 95-112. 
[17] P. Varkonyi, D. Tikk, P. Korondi, P. Baranyi, A New Algorithm for RNO-INO Type Tensor Product Model Representation, 2005 IEEE International Conference on Intelligent Engineering Systems, Cruising on the Mediterranean Sea, Spain, 2005, pp. 263-266.

doi: https://doi.org/10.1109/INES.2005.1555170

[18] Y. Yam, P. Baranyi, C.T. Yamg, Reduction of Fuzzy Rule Base via Singular Value Decomposition, IEEE Transactions on Fuzzy Systems 7 (2) (1999) pp. 120-132.

doi: https://doi.org/10.1109/91.755394

[19] G. Bergqvist, E. Larsson,The Higher Order Singular Value Decomposition: Theory and an Application, IEEE Signal Processing Magazine 27 (3) (2010) pp. $151-154$.

doi: https://doi.org/10.1109/MSP.2010.936030

[20] P. Baranyi, P. Szeidl, P. Várlaki, Y. Yam, Definition of the HOSVD Based Canonical Form of Polytopic Dynamic Models, Proceedings of the 2006 IEEE International Conference on Mechatronics, Budapest, 2006 pp. 660-665. doi: https://doi.org/10.1109/ICMECH.2006.252604

[21] P. Szeidl, P. Várlaki, HOSVD Based Canonical Form for Polytopic Models of Dynamic Systems, Journal of Advanced Computational Intelligence and Intelligent Informatics 13 (1) (2009) pp. 52-60.

[22] M. Kuczmann, Signals and Systems, Universitas- Győr Nonprofit Kft., Győr, 2010.

[23] V. C. S. Campos, F. O. Souza, L. A. B. Torres, R. M. Palhares, New Stability Conditions Based on Piecewise Fuzzy Lyapunov Functions and Tensor Product Transformations, IEEE Transactions on Fuzzy Systems 21 (4) (2013) pp. 784 760.

doi: https://doi.org/10.1109/TFUZZ.2012.2230178

[24] H. O. Wang, K. Tanaka, M. F. Griffin, Parallel Distributed Compensation of Nonlinear Systems by Takagi-Sugeno Fuzzy Model, Proceedings of 1995 IEEE International Conference on Fuzzy Systems, Yokohama, Japan, 1995 pp. 531538.

doi: https://doi.org/10.1109/FUZZY.1995.409737 
[25] B. P. Lal, T. Barjeev, O. G. Hari, Optimal Control of Nonlinear Inverted Pendulum System using PID Controller and LQR: Performance Analysis without and with Disturbance Input, International Journal of Automation and Computing (2014) pp. 661-670.

[26] M. Kuczmann, Comprehensive Survey of PID Controller Design for the Inverted Pendulum, Acta Technica Jaurinensis 12 (1) (2019) pp. 55-81. doi: https://doi.org/10.14513/actatechjaur.v12.n1.492

[27] G. Zhao, Z. Wang, Z. Song, A Novel Tensor Product Model Transformationbased Adaptive Variable Universe of Discourse Controller, Journal of the Franklin Institute 353 (17) (2016) pp. 4471-4499.

doi: https://doi.org/10.1016/j.jfranklin.2016.08.026

[28] S. Kuntanapreeda, Tensor Product Model Transformation Based Control and Synchronization of a Class of Fractional-Order Chaotic Systems, Asian Journal of Control 17 (2) (2015) pp. 371-380.

doi: https://doi.org/10.1002/asjc.839

[29] B. Lantos, Theory and Design of Control Systems I., Academic Press, Budapest, 2009.

[30] Z. Petres, B. Reskó, P. Baranyi, TP Model Transformation Based Control of the TORA System, Production Systems and Information Engineering (2004) pp. $159-175$.

[31] K. Tanaka, H. O. Wang, Fuzzy Control Systems Design and Analysis: A linear Matrix Inequality Approach, John Wiley and Sons, 2001.

doi: https://doi.org//10.1002/0471224596.ch2

[32] M. Kuczmann, State Space Based Linear Controller Design for the Inverted Pendulum, Acta Technica Jaurinensis 12 (2) (2019) pp. 130-147.

doi: https://doi.org/10.14513/actatechjaur.v12.n2.499 